\section{Accelerated Cutaneous Nodulosis Associated with Aromatase Inhibitor Therapy in a Patient with Rheumatoid Arthritis}

\section{To the Editor:}

Aromatase inhibitors have become widely used for the treatment of breast cancer. We describe a patient with rheumatoid arthritis (RA) treated with the aromatase inhibitor letrozole who subsequently developed accelerated cutaneous nodulosis.

A 71-year-old woman with a 21-year history of RA had established care at the University of California San Diego 7 years after her initial diagnosis, where she was noted to have a symmetric inflammatory arthritis of the metacarpophalangeal and proximal interphalangeal joints, wrists, elbows, and knees with an elevated titer of rheumatoid factor (RF) and anti-cyclic citrullinated antibody. She was given sulfasalazine and eventually methotrexate (MTX). However, because nodules appeared on the fingers and pressure areas such as the Achilles tendon, leflunomide was substituted for MTX. This was eventually discontinued due to inactive disease.

In December 2005, when she was no longer taking MTX or leflunomide, a chest radiograph disclosed lesions in the breast and lung suggestive of metastatic breast cancer. In March 2006, she started the aromatase inhibitor letrozole $2.5 \mathrm{mg}$ daily. In April 2006, a mastectomy and sentinel node biopsy revealed an invasive, mixed ductal and lobular carcinoma.

In June 2007 she reported the appearance of multiple small subcutaneous nodules on the fingers of both hands (Figure 1). She had minimal joint stiffness or pain. A diagnosis of "accelerated cutaneous nodulosis" was considered and because of a possible relationship to letrozole, the drug was discontinued. By November 2007, the number of nodules remained about the same, but they were smaller and less painful. Followup in May 2008 demonstrated continued decrease in size and tenderness of the nodules.

Aromatase inhibitors have become widely used in the treatment of estrogen-receptor-positive breast cancers. They markedly suppress plasma estrogen concentrations in postmenopausal women by inhibiting or inactivating aromatase, the enzyme responsible for the synthesis of estrogens from androgenic substrates.

Aromatase inhibitors have been associated with various rheumatologic syndromes. The most common reported in the literature is a musculoskeletal pain syndrome ${ }^{1}$. There has also been a case report of a patient developing RA after starting exemestane ${ }^{2}$. We believe our patient is the first reported case of an aromatase inhibitor possibly inducing accelerated cutaneous nodulosis. Of interest, there was no simultaneous worsening of joint symptoms from longstanding RA. Although prior treatment with antirheumatic therapy could account for her quiescent joint disease, this is in accord with observations that rheumatoid nodule activity is often not correlated with joint disease activity ${ }^{3}$

Histopathologically, the mature rheumatoid nodule is characterized by a central area of "fibrinoid" necrosis rimmed by fibroblasts and macrophages. This palisade is surrounded by a collagenous capsule with perivascular collections of chronic inflammatory cells, primarily macrophages and $\mathrm{T}$ lymphocytes.

The exact mechanisms underlying rheumatoid nodule formation are unknown ${ }^{3}$. Vasculitis has been suggested as the inciting lesion. Kato, et al demonstrated complexes of IgM RF and terminal complement components on some of the damaged vascular endothelial cells in rheumatoid nodules ${ }^{4}$. Cytokine analysis revealed expression of interferon- $\gamma$ (IFN- $\gamma$ ), interleukin $1 \beta$ (IL-1ß), and tumor necrosis factor- $\alpha$ (TNF- $\alpha$ ), and the absence of IL-2, IL-4, and IL-5, which suggests a Th1 profile similar to that of the synovi$u^{5}$. The cell adhesion molecule E-selectin has also been found to be increased in the rheumatoid nodule compared to the synovium ${ }^{6}$. Such data suggest that, as in the synovium, proinflammatory cytokines and other cell-signaling mechanisms play an important role in the pathogenesis of rheumatoid nodules.

The mechanism by which aromatase inhibitors might induce accelerated cutaneous nodulosis could be explained by estrogen's various immunomodulating capabilities. In rodents, for instance, estradiol (E2) directly influences dendritic cells and macrophages, innate and adaptive immune responses, B cell function, inflammatory cytokines, and the polarization of Th1/Th2 cells ${ }^{7,8}$. E2 could either potentiate Th1 proinflammatory responses by stimulating IL-12, TNF- $\alpha$, and IFN- $\gamma$ synthesis, or stimulate Th2 antiinflammatory cytokines such as IL-4, IL-10, and transforming growth factor- $\beta$.

One possibility is that aromatase inhibitors could shift the Th1/Th2 balance toward a humoral immune response and favor immune complex vasculitis. There have been 2 case reports of patients who developed a small-vessel cutaneous vasculitis while taking aromatase inhibitors 8,9 . Biopsies showed IgA deposits in the dermal vessels in one case, and complement components and $\operatorname{IgA}$ and $\operatorname{IgM}$ deposits in the other.

Alternatively, estrogen deprivation resulting from aromatase inhibition might skew cytokine expression further toward Th1-mediated tissue dam-

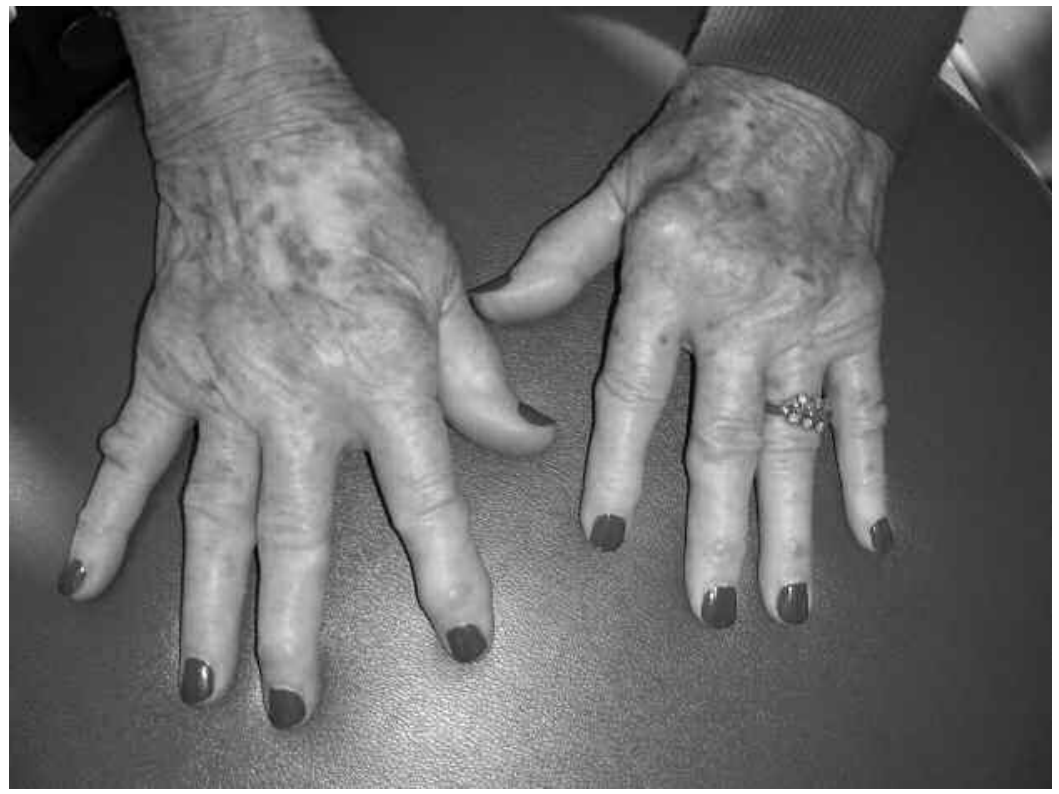

Figure 1. Multiple subcutaneous nodules on the hands of a patient after treatment with the aromatase inhibitor letrozole. 
age. As noted, the RA nodule is a granuloma and has a Th1 cytokine profile. In the case of this patient, one might expect that her joint symptoms would have worsened with the aromatase inhibitor treatment, but longstanding suppression of arthritis could account for quiescent joint symptoms.

Estrogen may also induce accelerated cutaneous nodulosis by altering cell adhesion. As noted, rheumatoid nodules have significantly more E-selectin expression compared to the synovium. In a rat model of endotoxin-induced uveitis, treatment with $17-\beta$-estradiol reduced expression of E-selectin ${ }^{10}$. It is possible that estrogen deprivation increases endothelial expression of E-selectin, leading to enhanced migration of inflammatory cells into the rheumatoid nodule, thus contributing to accelerated cutaneous nodulosis.

Based on our experience with this patient, physicians should be alerted to the possibility that accelerated cutaneous nodulosis might develop if aromatase inhibitors are used in patients with RA. Prospective immunological studies of such patients could improve our understanding of the effects of hormonal therapy on RA and its extraarticular manifestations.

JEANNIE CHAO, MD, University of California San Diego School of Medicine, La Jolla; BARBARA A. PARKER, MD, Moores UCSD Cancer Center, La Jolla; NATHAN J. ZVAIFLER, MD, University of California San Diego School of Medicine, 9500 Gilman Drive, La Jolla, California 92093, USA. Address reprint requests to Dr. Chao; E-mail: J5Chao@ucsd.edu

\section{REFERENCES}

1. Baum M, Buddzar AU, Cuzick J, et al, and the ATAC Trialists' Group. Anastrozole alone or in combination with tamoxifen versus tamoxifen alone for adjuvant treatment of postmenopausal women with early breast cancer: first results of the ATAC randomized trial.
Lancet 2002;359:231-9.

2. Morel B, Marotte H, Miossec P. Will steroidal aromatase inhibitors induce rheumatoid arthritis? [letter]. Ann Rheum Dis 2007;66:557-8.

3. Highton J, Hessian PA, Stamp L. The rheumatoid nodule: peripheral or central to rheumatoid arthritis? Rheumatology (Oxford) 2007;46:1385-7.

4. Kato H, Yamakawa M, Ogino T. Complement mediated vascular endothelial injury in rheumatoid nodules: a histopathological and immunohistochemical study. J Rheumatol 2000;27:1839-47.

5. Hessian PA, Highton J, Kean A, Sun CK, Chin M. Cytokine profile of the rheumatoid nodule suggests that it is a Th1 granuloma. Arthritis Rheum 2003;48:334-8.

6. Wikaningrum R, Highton J, Parker A, et al. Pathogenic mechanisms in the rheumatoid nodule. Arthritis Rheum 1998;41:1783-97.

7. Cutolo M, Capellino S, Sulli A, et al. Estrogens and autoimmune diseases. Ann NY Acad Sci 2006;1089:538-47.

8. Conti-Beltraminelli M, Pagani O, Ballerini G, et al. Henoch-Schönlein purpura during treatment with anastrozole [letter]. Ann Oncol 2007;18:205-7.

9. Shoda H, Inokuma S, Yajima N, Tnaka Y, Setoguchi K. Cutaneous vasculitis developed in a patient with breast cancer undergoing aromatase inhibitor treatment [letter]. Ann Rheum Dis 2005;64:651-2.

10. Miyamoto N, Mandai M, Suzuma I, Suzuma K, Kobayashi K, Honda Y. Estrogen protects against cellular infiltration by reducing the expressions of E-selectin and IL-6 in endotoxin-induced uveitis. J Immunol 1999;163:374-9.

J Rheumatol 2009;36:5; doi:10.3899/jrheum.080648 